\title{
Prospective, randomized, blinded, and placebo-controlled study of Cerebrolysin dose-response effects on long-term functional outcomes in a rat model of mild traumatic brain injury
}

\author{
Yanlu Zhang, MD, MS, ${ }^{1}$ Michael Chopp, PhD, ${ }^{2,3}$ Zheng Gang Zhang, MD, PhD, ${ }^{2}$ Yi Zhang, PhD, ${ }^{2}$ \\ Li Zhang, MD, ${ }^{2}$ Mei Lu, PhD, ${ }^{4}$ Talan Zhang, MS, ${ }^{4}$ Stefan Winter, PhD, ${ }^{5}$ Hemma Brandstätter, PhD, ${ }^{5}$ \\ Asim Mahmood, MD, ${ }^{1}$ and Ye Xiong, MD, PhD' 1
}

Departments of ${ }^{1}$ Neurosurgery, ${ }^{2}$ Neurology, and ${ }^{4}$ Biostatistics and Research Epidemiology, Henry Ford Hospital, Detroit, Michigan; ${ }^{3}$ Department of Physics, Oakland University, Rochester, Michigan; and ${ }^{5}$ EVER Pharma $\mathrm{GmbH}$, Unterach, Austria

\begin{abstract}
OBJECTIVE Cerebrolysin is a neuropeptide preparation that mimics the properties of neurotrophic factors and has had beneficial effects in the treatment of neurodegenerative diseases, stroke, and traumatic brain injury (TBI). To further evaluate treatment schemes, the authors assessed the dose-response of Cerebrolysin on functional improvement in a rat model of mild TBI (mTBI).
\end{abstract}

METHODS This dose-response study was a prospective, randomized, blinded, and placebo-controlled preclinical experiment. Male Wistar adult rats, subjected to mTBI induced by a closed head impact, were treated randomly with 0 (saline as placebo), $0.8,2.5$, or $7.5 \mathrm{ml} / \mathrm{kg}$ of Cerebrolysin 4 hours after mTBI and daily for a total of 10 consecutive days. A battery of cognitive and sensorimotor functional tests was performed over 90 days.

RESULTS The primary outcome was functional improvement over the 90 days; animal weight and death were the secondary and safety outcomes, respectively. A significant $(p<0.001)$ dose effect of Cerebrolysin on cognitive recovery 3 months after injury was found. Cerebrolysin at a dose of $\geq 0.8 \mathrm{ml} / \mathrm{kg}$ significantly $(p<0.001)$ improved cognitive outcome. The higher dose $(7.5 \mathrm{ml} / \mathrm{kg})$ resulted in significantly better cognitive recovery than the lowest doses $(0.8 \mathrm{ml} /$ $\mathrm{kg}$ ) but not relative to the $2.5-\mathrm{ml} / \mathrm{kg}$ dose. Cerebrolysin at a dose of 2.5 or $7.5 \mathrm{ml} / \mathrm{kg}$ also caused different onset times of significant improvement in sensorimotor function. No differences in body weight or mortality rate among the groups were found.

CONCLUSIONS This preclinical randomized, placebo-controlled, and blinded study with a clinically relevant treatment scheme revealed that Cerebrolysin at doses of $0.8-7.5 \mathrm{ml} / \mathrm{kg}$, administered 4 hours after $\mathrm{mTBI}$ and then once daily for a total of 10 consecutive days, improved functional outcomes 3 months after injury. A dose of $2.5 \mathrm{ml} / \mathrm{kg}$ is likely an optimal dose for the treatment of experimental mTBI.

https://thejns.org/doi/abs/10.3171/2017.6.JNS171007

KEY WORDS Cerebrolysin; cognitive recovery; dose-response; mild traumatic brain injury; neurological outcome

$\mathrm{T}$ RAUMATIC brain injury (TBI) is one of the leading causes of death and morbidity globally. The majority of more than 1.7 million TBIs in the United States each year are diagnosed as mild TBI (mTBI). ${ }^{12}$ Some people with mTBI have a measurable cognitive deficit 1 year later ${ }^{45}$ even though no obvious brain tissue damage can be detected.$^{19,52}$ No effective treatment for TBI exists because all Phase II/III TBI clinical trials have failed. ${ }^{28,35}$ Clinical trials for TBI involve mainly moderate to severe injury. ${ }^{36}$
No effective, standard pharmacological treatment currently exists specifically for cognitive symptoms of patients with mTBI. Considering the high incidence of mTBI and associated cognitive deficits, ${ }^{14}$ the development of effective treatments for mTBI is an unmet medical need.

Cerebrolysin (EVER Pharma) is a low-molecularweight neuropeptide preparation obtained through standardized enzymatic proteolysis of brain proteins and exhibits neuroprotective and neurotrophic properties similar

ABBREVIATIONS mNSS = modified neurological severity score; $\mathrm{mTBI}=$ mild TBI; MWM = Morris water maze; NOR = novel object recognition; RCT = randomized controlled trial; $\mathrm{TBI}=$ traumatic brain injury.

SUBMITTED April 21, 2017. ACCEPTED June 20, 2017.

INCLUDE WHEN CITING Published online January 5, 2018; DOI: 10.3171/2017.6.JNS171007. 
to those that occur naturally in neurotrophic growth factors. ${ }^{46}$ In previous studies, it significantly decreased the levels of tau phosphorylation by regulating kinase activity in a mouse Alzheimer disease model with amyloid precursor protein overexpression ${ }^{54}$ and improved functional outcome by increasing neurogenesis in a rat stroke model..$^{58}$ Cerebrolysin improved functional recovery in a rat stabwound TBI model by reducing brain edema when it was administered 5-60 minutes after injury. ${ }^{49}$ Recent clinical trials on stroke, TBI, and Alzheimer disease found that Cerebrolysin is safe and beneficial. ${ }^{1,6,42,43}$ Cerebrolysin falls into the category of drugs that have been approved in countries outside of the United States mainly for treatment of cognitive impairment in dementia, stroke, and TBI and mainly in Europe, Asia, and South America. Cerebrolysin administration in patients with severe disability after TBI is associated with a decreased mortality rate and improved favorable outcome (i.e., 3- and 6-month Glasgow Outcome Scale-Extended scores). ${ }^{31}$ Cerebrolysin improved the cognitive function, especially long-term memory, of patients with mTBI 3 months after injury in a double-blind, placebo-controlled, randomized study. ${ }^{8}$ Therefore, Cerebrolysin might be a promising therapy for TBI.

Cerebrolysin is used currently outside the United States for the treatment of human TBI. However, the optimal dosing strategy in the context of different severities of TBI needs further evaluation. The current choice of the clinical dose for Cerebrolysin in patients with mTBI might be suboptimal. Among many other factors, suboptimal dosing has been considered a possible factor in the negative Phase III clinical trial results of treatments for TBI that include progesterone. ${ }^{27}$ Therefore, to advance our knowledge of Cerebrolysin administration for the treatment of TBI with the intention to optimize protocols for future clinical studies, we designed a dose-response study in rats subjected to mTBI. To maximize translational power, we followed current guidelines on the design and reporting of experimental animal studies ${ }^{32}$ for this prospective, randomized, blinded, and placebo-controlled preclinical trial. Considering the high incidence of mTBI and the lack of effective treatments, we used a Marmarou impact-acceleration rat model of diffuse $\mathrm{TBI}^{4,17,40}$ to induce clinically relevant mild closed head injury and to determine the dose-response of Cerebrolysin on long-term cognitive and sensorimotor functional recovery.

\section{Methods}

All experimental procedures were carried out in accordance with the National Institutes of Health Guide for the Care and Use of Laboratory Animals and were approved by the Institutional Animal Care and Use Committee of Henry Ford Hospital.

\section{Design and Oversight}

This dose-response study was a prospective, randomized, placebo-controlled, and double-blinded end-point study. Adult Wistar rats subjected to mTBI were assigned randomly to receive 1 of 4 Cerebrolysin doses $(0,0.8,2.5$, or $7.5 \mathrm{ml} / \mathrm{kg}$ ) initiated 4 hours after injury and continued daily for a total of 10 consecutive days. The doses of
Cerebrolysin were selected based on our previous studies in rats with experimental stroke ${ }^{59}$ or mTBI. ${ }^{60}$ The study design, surgeries, preparation of study medication, treatment, data collection, and analysis were each performed by different and independent members of the Henry Ford Hospital. Persons who performed experiments or outcome assessments were blinded to the treatment. EVER Pharma GmbH supplied Cerebrolysin, opaque syringes, and a grant to support study infrastructure but was not involved in conducting the study, data collection, or data analysis.

\section{Study Animals and mTBI Model}

In our experiments, we used 3- to 4-month-old male Wistar rats (Charles River Breeding Company) with a mean ( \pm SD) weight of $417 \pm 30 \mathrm{~g}$ (range 326-471 g). After 1 week of quarantine and acclimation, the rats were anesthetized initially with $4 \%$ isoflurane and maintained throughout the surgical period with $1.0 \%-1.5 \%$ isoflurane in $70 \% \mathrm{~N}_{2} \mathrm{O}$ and $30 \% \mathrm{O}_{2}$ via a nose mask. Their rectal temperature was maintained at a mean of $37^{\circ} \mathrm{C} \pm 0.5^{\circ} \mathrm{C}$ (range $36.1^{\circ} \mathrm{C}-37.6^{\circ} \mathrm{C}$ ) during the surgery. The surgeries were performed and mTBIs inflicted in accordance with the procedure described in detail in our previous study report. ${ }^{60}$ In brief, a $2-\mathrm{cm}$ midline incision was made using a scalpel, and the skull was exposed. To prevent skull fracture, a small stainless steel helmet-disk was placed on the rodent's skull while the animal was supported by a foam bed. Closed head injury was induced by dropping a cylindrical column of segmented brass $(450 \mathrm{~g})$ through a Plexiglas tube from a distance $(1 \mathrm{~m})$ onto the disk fixed to the skull vault of the animal. The incision was closed with sterile 4-0 sutures. Rats that died on impact and those with a skull fracture were excluded from the study.

To assess acute injury effects, apnea and times to toepinch, tail-pinch, and self-righting reflexes were all monitored immediately after each injury. The self-righting reflex represents the time the animal takes to right itself from a supine position to a prone position with all 4 paws against a table surface. A delay in self-righting reflex reflects transient unconsciousness. ${ }^{29}$ The loss of self-righting reflex in animals after TBI is considered analogous to loss of consciousness in humans after TBI and can be considered a behavioral indicator of injury severity. ${ }^{14,22}$

\section{Study Design and Treatment Regimen}

Animals were assigned randomly in a 1:1:1:1 allocation to receive saline or Cerebrolysin at a dose of $0,0.8,2.5$, or $7.5 \mathrm{ml} / \mathrm{kg}$ (equivalent to human doses of $0,0.13,0.42$, and $1.25 \mathrm{ml} / \mathrm{kg}$, respectively) via intraperitoneal injection..$^{55}$ Randomization schema were generated using nQuery 3.0. The randomization was based on a mixed block size. Study medication was prepared by a designated investigator (who was not involved in any other part of this study), according to animal body weight, 1 day before treatment based on the randomization scheme ( $\mathrm{n}=12$ per group, as planned). All experimental drugs were prepared in opaque syringes at a constant volume of $3 \mathrm{ml}$ (adjusted with saline) and administered at scheduled injection times. The first treatment was administered 4 hours after the mTBI and repeated once daily for a total of 10 consecutive days. Laboratory 


\begin{tabular}{|c|c|c|c|c|c|c|c|}
\hline \multirow[b]{2}{*}{ Test } & \multicolumn{7}{|c|}{ Time After mTB|* } \\
\hline & 1 Day & 7 Days & 14 Days & 21 Days & $1 \mathrm{Mo}$ & 2 Mos & 3 Mos \\
\hline Sensorimotor functional recovery (mNSS \& foot-fault \& adhesive-removal tests) & $X$ & $X$ & $X$ & $X$ & $\mathrm{X}$ & $X$ & $\mathrm{X}$ \\
\hline \multicolumn{8}{|l|}{ Cognitive recovery } \\
\hline $\begin{array}{l}\text { Cognition (MWM test), latency (secs), time in correct quadrant (\%), \& swim speed } \\
(\mathrm{m} / \mathrm{sec})\end{array}$ & & & & & & & $\mathrm{X}$ \\
\hline Social interaction test & & & & & $\mathrm{X}$ & & $\mathrm{X}$ \\
\hline NOR test & & & & & $\mathrm{X}$ & & $\mathrm{X}$ \\
\hline
\end{tabular}

personnel who inflicted the mTBI and performed drug injections and outcome assessment were blinded to the treatment for each animal. The animals were monitored closely for any adverse events after they were enrolled.

Animals were assigned randomly into 1 of 5 groups: the sham-surgery (age-matched control) group or 1 of 4 treatment groups, which received a Cerebrolysin dose of 0 (vehicle), $0.8,2.5$, or $7.5 \mathrm{ml} / \mathrm{kg}$. Cerebrolysin was administered intraperitoneally to the rats daily for 10 days, starting 4 hours after the mTBI. Injured animals treated with a $0-\mathrm{ml} / \mathrm{kg}$ dose of Cerebrolysin (saline) were used as a treatment control group. An age-matched control sham group (that is, with surgery but without injury and treatment) was included as the reference group. Acute neurological assessments (i.e., toe-pinch, tail-pinch, and self-righting reflexes) were monitored before study treatment, immediately after the mTBI, or, in the sham-surgery group, every 10 seconds until the animals were able to self-right. Four sets of behavioral tests (i.e., Morris water maze [MWM], 3-chamber social interaction, novel object recognition [NOR], and sensorimotor function [modified neurological severity score ${ }^{38}$ and foot-fault and adhesive-removal tests]) were performed on all animals at various time points over the 3 months (Table 1). The MWM tests were performed only at month 3, for the last 5 days, and the average of the last 3-day MWM test scores was used for the analysis. In addition, the MWM test has 3 components (as listed in Table 1). MWM swim speed was used as the reference measure; we did not expect any differences in these average scores among all the mTBI groups. This test was excluded from the end-point analysis.

\section{Study Outcomes}

We used the following 2 primary end points: 1) cognitive function, which was measured by the MWM, social interaction, and NOR tests, and 2) sensorimotor function, which was measured by the modified neurological severity score (mNSS) and the foot-fault and adhesive-removal tests. The secondary end points are cognitive recovery or sensorimotor functional recovery at various time points, before 3 months after mTBI. A TBI worsening, death, and weight change were used as safety end points.

\section{Adhesive Patch-Removal Test}

Two pieces of adhesive-backed paper $\left(113.1 \mathrm{~mm}^{2}\right)$ were used as bilateral tactile stimuli occupying the distal-radial region on the wrist of each forelimb.$^{50}$ Each animal underwent 3 trials per testing day, and the mean time (in sec- onds) required to remove the stimuli from each forelimb was recorded. The average of the mean times for both forelimbs was used for statistical analysis.

\section{Foot-Fault Test}

Each rat was tested for placement dysfunction of the forelimbs with the modified foot-fault test. ${ }^{25}$ The rat was placed on a horizontal grid. A foot fault was noted when a paw fell through an opening in the grid floor. The total number of steps (movement of each paw) that the rat used to cross the grid and the total number of foot faults (falls or slips between the wires) for the paw were recorded. Data are presented as percentages of paw foot faults.

\section{mNSS}

The mNSS is a composite of motor, sensory, reflex, and balance test scores. Neurological function was graded on a scale of 0 (normal) to 18 (maximal deficit), as previously described. ${ }^{9}$

\section{MWM Test}

The modified MWM test was used to assess spatial learning function, as previously described. ${ }^{10,61}$ This test was performed daily for 5 days on all rats 3 months after injury (i.e., on days 86-90) (for details, see Supplemental Information).

\section{NOR Test}

The NOR task is a well-characterized behavioral measure of hippocampally based working nonspatial visual recognition memory in rodents..$^{51}$ It can be completed in a short time so the animals do not feel stressed, and it can assess recognition memory after only 1 trial, which is an advantage over other methods. ${ }^{5}$ This test was performed on all rats 1 and 3 months after injury (for details, see Supplemental Information).

\section{Three-Chamber Social Interaction Test}

This test is used to assess memory for interactions with novel conspecifics. ${ }^{11,21}$ Rats tend to spend more time interacting with a novel rat versus one they have encountered previously. The 3-chamber test can help identify rodents with deficits in sociability and/or social novelty. ${ }^{21} \mathrm{~A}$ decreased duration and/or number of contacts can be associated with depressive and/or anxiety-like behaviors ${ }^{48}$ which are common after TBI, especially after mTBI or with post- 
TABLE 2. Acute response assessment according to reflex after mTBI

\begin{tabular}{lccc}
\hline \multicolumn{1}{c}{ Group } & Toe-Pinch Reflex & Tail-Pinch Reflex & Self-Righting Reflex \\
\hline Sham $(n=12)$ & $12.25(1.10)$ & $40.00(2.95)$ & $68.50(3.25)$ \\
\hline $\mathrm{mTBI}+$ Cerebrolysin 0 $\mathrm{ml} / \mathrm{kg}(\mathrm{n}=12)$ & $28.42(6.16)$ & $55.00(9.31)$ & $102.92(8.86)$ \\
\hline $\mathrm{mTBI}+$ Cerebrolysin 0.8 ml/kg $(\mathrm{n}=12)$ & $30.92(2.97)$ & $60.67(7.64)$ & $102.25(10.82)$ \\
\hline $\mathrm{mTBI}+$ Cerebrolysin $2.5 \mathrm{ml} / \mathrm{kg}(\mathrm{n}=12)$ & $29.92(6.21)$ & $57.67(7.05)$ & $103.50(11.44)$ \\
\hline $\mathrm{mTBI}+$ Cerebrolysin $7.5 \mathrm{ml} / \mathrm{kg}(\mathrm{n}=12)$ & $38.83(7.58)$ & $62.42(9.55)$ & $124.75(14.43)$ \\
\hline
\end{tabular}

Values are presented as mean (SE) times in seconds.

traumatic stress disorder. ${ }^{16}$ This test was performed on all rats 1 and 3 months after injury (for details, see Supplemental Information).

\section{Statistical Analysis}

A baseline balance was checked for acute neurological response using the Wilcoxon test, and if any variable in the baseline was not balanced, it was used as a covariate for adjustment of the outcomes. The sham-surgery group was used as the reference when we tested the mTBI deficits. For dose analysis, the sham-surgery group was excluded. Data were evaluated for normality; ranked data or the nonparametric Kruskal-Wallis test was used when data were not distributed normally.

The global test using generalized estimating equations $\mathrm{s}^{37}$ was used to analyze the dose effect on the 2 primary end points, measured from multiple tests. A Bonferroni correction $\alpha$ value of 0.025 was used to test the dose effect for 2 end points. A dose effect was detected if there was a significant difference at an end point with a $p$ value of $\leq 0.025$ compared with controls with a dose of $0 \mathrm{ml} / \mathrm{kg}$. For each end point, the analysis began by testing the overall dose effect at the criterion ( $\alpha$ value) of $<0.025$ with estimates of means among dose groups. A pairwise dose comparison would follow if an overall dose effect was observed, or otherwise as an exploratory analysis. A negative value of mean difference (e.g., dose $0.8 \mathrm{vs} 0 \mathrm{ml} / \mathrm{kg}$ ) with $\mathrm{p}<$ 0.025 indicates a significant dose effect on reduction of the worst outcome. The individual outcome test was also conducted if we found an overall dose effect. A mixed model was used for longitudinal outcomes considering a possible time-by-dose interaction. A similar analysis was used to study the dose effect on weight as a safety end point. All analyses were performed using SAS 9.4.

An effective dose was determined to be the lowest dose that significantly improves the functional/behavioral recovery more than in the control group 3 months after injury.

\section{Results}

A total of 60 animals were included in the study, and they were assigned randomly into 1 of 5 groups, including a sham-surgery group and 4 groups of rats with mTBI and treated with 0 (saline), $0.8,2.5$, or $7.5 \mathrm{ml} / \mathrm{kg}$ of Cerebrolysin; 12 animals were included in each group. No skull fractures or apneas were observed immediately after impact. No animal died after the mTBI was inflicted or during the 3-month study period.

\section{Acute Neurological Assessments at Baseline (after injury)}

Results of the combined mTBI groups $(n=48)$ were compared with those of the sham-surgery group $(n=12)$. We observed significant deficits in all 3 acute outcomes in the mTBI groups compared with the sham-surgery group (toe-pinch reflex, $\mathrm{p}<0.001$; self-righting reflex, $\mathrm{p}<0.001$; tail-pinch reflex, $p=0.039$ ). The mean (SE) times for each group are presented in Table 2.

\section{Injury-Severity Balance at Baseline}

No difference in acute neurological responses was detected at baseline among the mTBI groups (Table 2). p values for the toe-pinch, tail-pinch, and self-righting reflexes were $0.614,0.929$, and 0.451 , respectively, among the 4 groups of rats after mTBI. The lack of significant differences in these acute neurological indices suggests that each rat in the mTBI groups experienced an injury of equivalent severity before study treatment.

\section{Primary End Points: Dose-Response Effect on Functional Recovery at the Month 3 Cognitive End Point}

The overall dose effect on cognitive recovery 3 months after mTBI, analyzed by the global test, was significant ( $\mathrm{p}$ $<0.0001$ ). Animals treated with Cerebrolysin 4 hours after $\mathrm{mTBI}$ at a dose of $0.8,2.5$, or $7.5 \mathrm{ml} / \mathrm{kg}$ showed significant improvement in cognitive outcomes compared with those of the control group. The $7.5-\mathrm{ml} / \mathrm{kg}$ dose resulted in significantly better outcomes than the $0.8-\mathrm{ml} / \mathrm{kg}$ dose. We found no significant difference between the 2.5- and 7.5$\mathrm{ml} / \mathrm{kg}$ doses in their effects on cognitive recovery. Results of the subgroup analysis after the global test indicated that Cerebrolysin at doses of $0.8,2.5$, or $7.5 \mathrm{ml} / \mathrm{kg}$ had significant effects on cognitive function test results compared with those of the control group 3 months after injury. For the MWM test, we observed no difference in swim speeds among all the groups $(\mathrm{p}=0.48)($ Fig. $1 \mathrm{~A})$, which indicates that mTBI did not affect the swim speed of injured rats and that the swim speed did not contribute to spatial learning and memory deficits in those rats. Compared with the control group, rats in the Cerebrolysin treatment group (at all 3 doses) spent increased time in the correct quadrant $(\mathrm{p}<0.05)$ (Fig. 1B) and took a significantly reduced time (latency) to reach the hidden platform in the MWM ( $\mathrm{p}<$ 0.05) (Fig. 1C). For the NOR test, significantly more time was spent exploring the novel object than the familiar object by rats with mTBI treated with Cerebrolysin at any of the 3 doses 1 and 3 months after injury than by the control group (p < 0.05) (Fig. 2A and B). Similarly, for social in- 

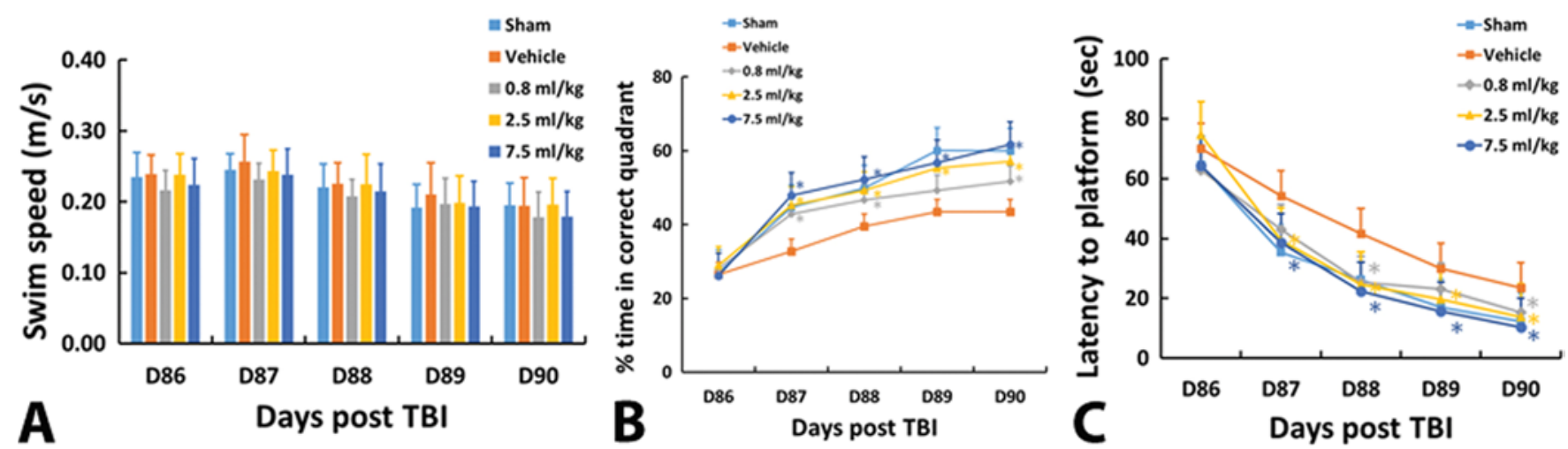

FIG. 1. Effects of Cerebrolysin on spatial learning performance in the MWM test 3 months after mTBI. A: mTBI did not affect the swim speed of injured rats compared with sham-surgery-group rats. There was no significant difference in the swim speed of Cerebrolysin-treated rats compared with saline-treated rats. B: Cerebrolysin treatment significantly improved spatial learning performance compared with that of the vehicle group at 3 months after mTBI. C: Cerebrolysin treatment significantly reduced the time to reach the hidden platform in the MWM compared with that of the saline group at 3 months after mTBI. ${ }^{*} p<0.05$ versus vehicle (saline). Data represent means $( \pm S D) ; n=12$ rats per group. Figure is available in color online only.

teraction measured by the 3-chamber test, mTBI caused social interaction deficit 1 and 3 months after injury. Significantly more time was spent exploring the novel rat than the familiar rat by rats with mTBI treated with Cerebrolysin at any of the 3 doses 1 and 3 months after injury than by the control group $(\mathrm{p}<0.05)$ (Fig. 3A and B).

\section{Sensorimotor Functional Outcomes}

Different doses of Cerebrolysin resulted in diverse timings of onset of significant neurological improvement. On day 1 after mTBI, the overall dose effect was marginal ( $p$ $=0.05381)$, and the $2.5-\mathrm{ml} / \mathrm{kg}$ dose had a significant effect $(\mathrm{p}=0.00594)$. The subgroup analysis found that rats in the $2.5-\mathrm{ml} / \mathrm{kg}$ dose group experienced a significantly reduced frequency of foot-fault occurrences as measured by the foot-fault test compared with that in the control and the group that received a $0.8-\mathrm{ml} / \mathrm{kg}$ dose (Fig. 4A). On day 7 after mTBI, the overall dose effect was significant ( $\mathrm{p}$ $=0.02165)$. Rats in the $2.5-\mathrm{ml} / \mathrm{kg}$ dose group showed a significant effect compared with the control $(p=0.02386)$ and $0.8-\mathrm{ml} / \mathrm{kg}$ dose $(\mathrm{p}=0.02003)$ groups. Specifically, Cerebrolysin at a dose of $2.5 \mathrm{ml} / \mathrm{kg}$ significantly reduced the frequency of foot-fault occurrences and the time for removing adhesive from the forelimbs $(\mathrm{p}<0.05)$ (Fig. 4B). On day 14, no dose effect was observed. On day 21 , the overall dose effect was significant $(\mathrm{p}=0.00396)$. Rats in the $2.5-\mathrm{ml} / \mathrm{kg}$ group showed a significant effect compared with the control rats $(\mathrm{p}=0.04484)$ and those that received the $0.8-\mathrm{ml} / \mathrm{kg}$ dose $(\mathrm{p}=0.00037)$. One month after injury, the dose effect was not significant $(p=0.24558)$. Two months after injury, rats that received the $2.5-\mathrm{ml} / \mathrm{kg}$ dose had a significantly reduced time for adhesive removal compared with control rats $(\mathrm{p}=0.00789)$ and those that received the $0.8-\mathrm{ml} / \mathrm{kg}$ dose $(\mathrm{p}=0.01965)$, and the 7.5$\mathrm{ml} / \mathrm{kg}$ dose significantly reduced the adhesive-removal time $(\mathrm{p}=0.04455)$ and had a marginal effect on reducing the mNSS $(p=0.05020)$ over that of no treatment (control group). Three months after injury, the rats that received the $7.5-\mathrm{ml} / \mathrm{kg}$ dose had a significantly reduced adhesive-removal time compared with that of the controls $(\mathrm{p}=0.00506)$. Overall, Cerebrolysin at a dose of 2.5 or $7.5 \mathrm{ml} / \mathrm{kg}$ significantly reduced the adhesive-removal time over the 90 days, and the $2.5-\mathrm{ml} / \mathrm{kg}$ dose significantly reduced the frequency of foot-fault occurrences at early time
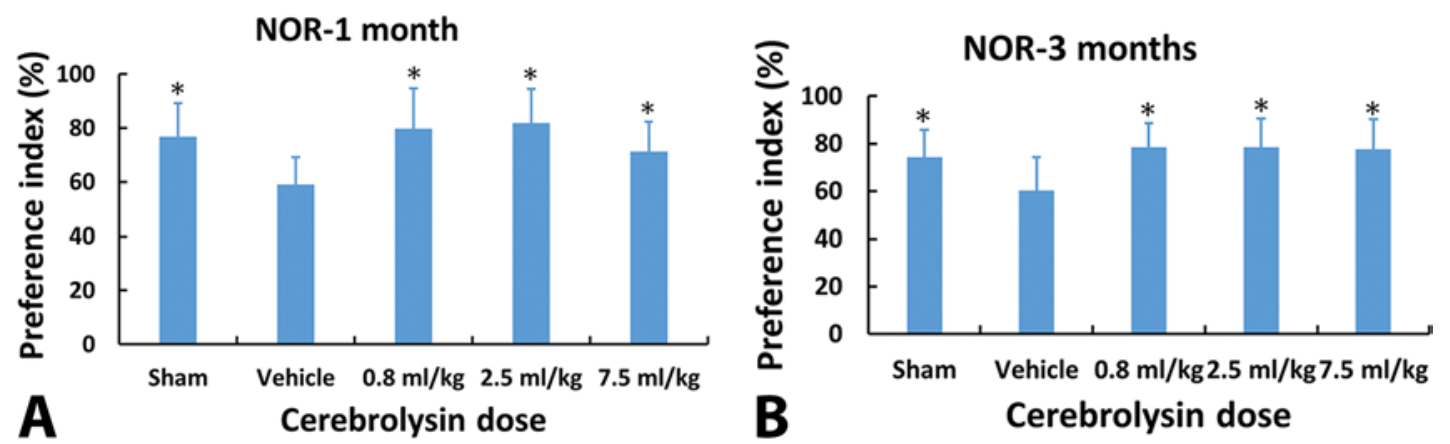

FIG. 2. Effects of Cerebrolysin on NOR memory 1 (A) and 3 (B) months after mTBI. Control rats spent significantly more time exploring the novel object than the familiar object. Rats with mTBI treated with vehicle showed impaired NOR memory 1 and 3 months after injury, whereas rats with $\mathrm{mTBI}$ treated with Cerebrolysin showed significantly improved NOR memory compared with the vehicle group $1(A)$ and $3(B)$ months after TTBI. $^{*} p<0.05$ versus vehicle. Data represent means $( \pm S D) ; n=12$ rats per group. Figure is available in color online only. 

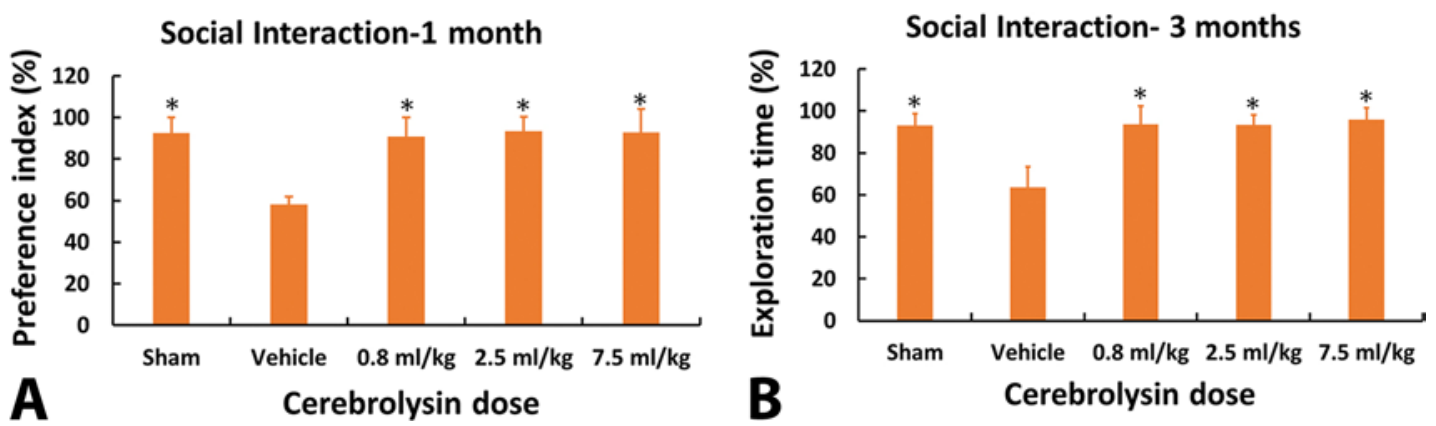

FIG. 3. Effects of Cerebrolysin on social interaction measured by the 3-chamber test 1 (A) and 3 (B) months after mTBI. Control rats spent significantly more time exploring the novel rat than the familiar rat. Rats with mTBI treated with vehicle spent equivalent times exploring the novel rat and the familiar rat 1 and 3 months after injury. Cerebrolysin treatment significantly improved social interaction compared with saline treatment $1(\mathrm{~A})$ and $3(\mathrm{~B})$ months after mTBI. ${ }^{*} p<0.05$ versus vehicle. Data represent means $( \pm$ $\mathrm{SD}) ; n=12$ rats per group. Figure is available in color online only.

points ( 1 and 7 days after injury). Cerebrolysin at both the 2.5 - and $7.5-\mathrm{ml} / \mathrm{kg}$ doses had a marginal effect on reducing the mNSS (Fig. 4C).

\section{Safety}

In general, all the animals gained weight over time (significant time effect) (Fig. 5). However, we found no doseby-time interaction $(\mathrm{p}=0.29)$ or effect on weight. The animals in each group gained weight over time $(\mathrm{p}<0.01)$. No other adverse effects of Cerebrolysin (i.e., death, seizures, dehydration, etc.), even at the highest dose $(7.5 \mathrm{ml} /$ $\mathrm{kg}$ ), were observed during the 90-day study, which is consistent with the safety profile for this agent in animals and humans. In this study, no deaths occurred. Weight gain was monitored as the only major safety index, in addition to multiple functional tests.

\section{Discussion}

\section{Study Outcomes}

In this prospective, randomized, blinded, placebocontrolled preclinical study, we explored the efficacy and safety of Cerebrolysin in the treatment of rats with mTBI.
Acute neurological responses were comparable among all the mTBI groups at baseline (immediately after mTBI), which indicates that all rats in the mTBI groups sustained an equivalent brain injury before therapeutic treatment. The rats with mTBI showed functional deficits at baseline and 3 months after mTBI compared with rats in the sham-surgery group. We found no effects of Cerebrolysin on mortality or safety parameters (animal body weight), which shows that the administration of Cerebrolysin doses up to $7.5 \mathrm{ml} / \mathrm{kg}$ was safe in animals with $\mathrm{mTBI}$. A dose of $2.5 \mathrm{ml} / \mathrm{kg}$ is likely optimal for the treatment of experimental rats with mTBI. Additional studies are warranted to assess whether more severe forms of TBI require altered dosing schemes.

The results of this study indicate a trend in the treatment effect of Cerebrolysin to gradually increase with dose; 7.5 $\mathrm{ml} / \mathrm{kg}$ is better than $0.8 \mathrm{ml} / \mathrm{kg}$. The highest dose we used was $7.5 \mathrm{ml} / \mathrm{kg}$, which is approximately equivalent to $90 \mathrm{ml}$ in humans; ${ }^{51,55}$ this dose has not been clinically tested in humans with TBI. Cerebrolysin at $0.8 \mathrm{ml} / \mathrm{kg}$ initiated 4 hours after mTBI significantly improved cognitive outcomes, and when administered at a higher dose $(2.5$ or $7.5 \mathrm{ml} / \mathrm{kg})$, it was also associated with faster recovery. A significant dif-

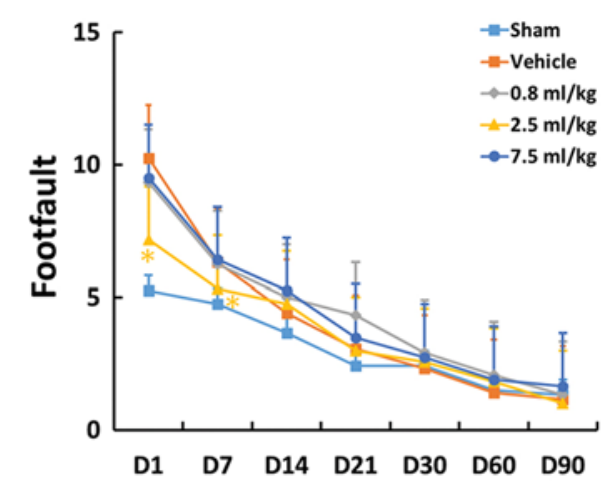

A

\section{Days post TBI}
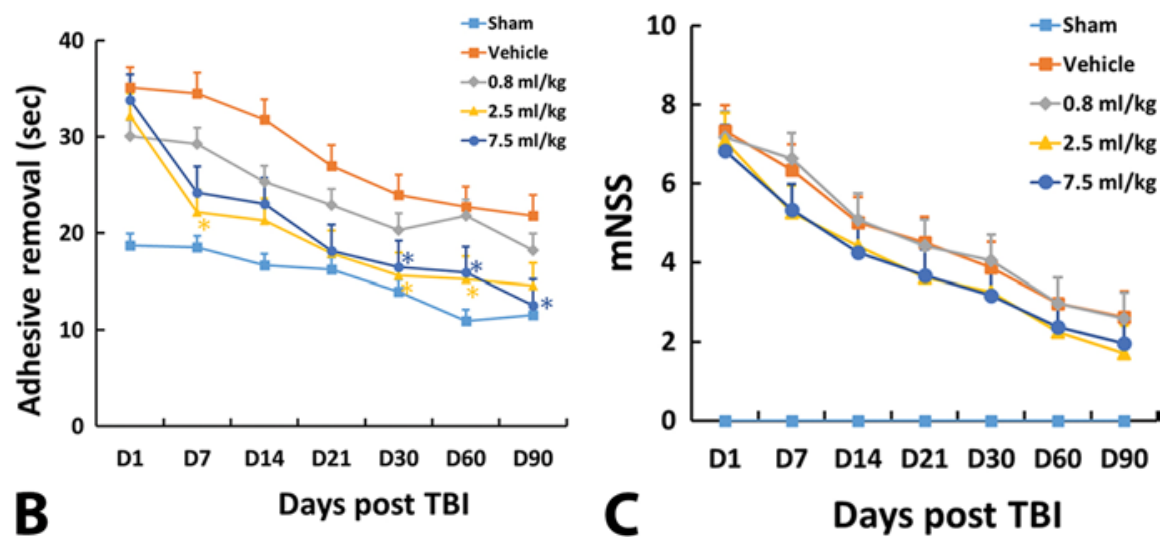

FIG. 4. Effects of Cerebrolysin on sensorimotor functional outcomes measured by the foot-fault (A) and adhesive-removal (B) tests and the $\mathrm{mNSS}(\mathrm{C})$. A: Cerebrolysin treatment at $2.5 \mathrm{ml} / \mathrm{kg}$ significantly reduced the frequency of foot faults from day 1 to 7 after $\mathrm{mTBI}$ compared with vehicle treatment. B: Cerebrolysin treatment at doses of 2.5 and $7.5 \mathrm{ml} / \mathrm{kg}$ resulted in significantly reduced adhesive-removal times compared with vehicle treatment. C: Cerebrolysin did not significantly lower the mNSSs compared with vehicle treatment. " $p<0.05$ versus vehicle. Data represent means $( \pm S D) ; n=12$ rats per group. Figure is available in color online only. 


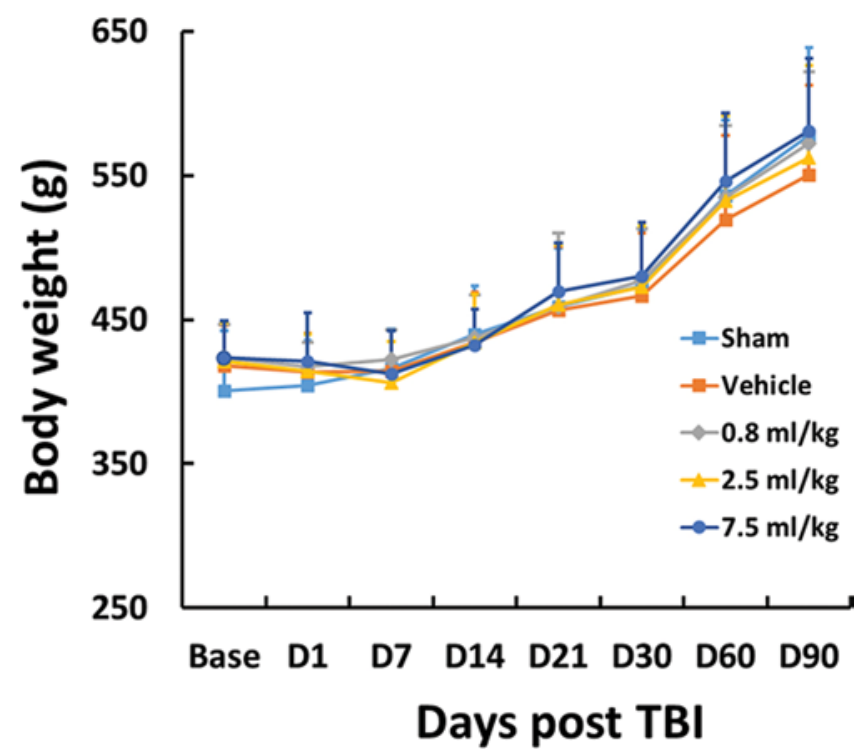

FIG. 5. Effects of mTBI and Cerebrolysin on body weight. We found no significant difference in the body weight of rats with mTBI treated with vehicle or Cerebrolysin compared with control rats. Data represent means $( \pm S D) ; n=12$ rats per group. Figure is available in color online only.

ference between the 0.8 - and $7.5-\mathrm{ml} / \mathrm{kg}$ doses was found in sensorimotor functional test results at some time points. However, the difference in cognitive outcomes of the rats in the 2.5 - and $7.5-\mathrm{ml} / \mathrm{kg}$ dose groups did not reach statistical significance. The $2.5-\mathrm{ml} / \mathrm{kg}$ dose is equivalent to the dose for humans $(0.4 \mathrm{ml} / \mathrm{kg}, 30-\mathrm{ml}$ infusion per day for humans with a 70- to $75-\mathrm{kg}$ body weight) commonly used to treat $\mathrm{TBI}^{8,42}$ and stroke. ${ }^{43}$ In our study, the $0.8-\mathrm{ml} / \mathrm{kg}$ dose (approximately equivalent to $10 \mathrm{ml} /$ day in an adult human) initiated 4 hours after injury in the rats also showed significant efficacy compared with saline treatment. Cerebrolysin administration (10-ml dosage started 1 month after injury and then once daily for a total of 30 days) is associated with functional recovery in patients with severe disability after TBI. ${ }^{31}$

\section{Study Strengths}

More than 30 clinical TBI trials for neuroprotection have failed to yield a therapeutic agent for clinical use. ${ }^{23,44}$ Many reasons for these failures have been proposed and reviewed. ${ }^{23,44}$ In addition to the tremendous heterogeneity of the population of humans with TBI and some limitations of animal TBI models, most of the preclinical trials for testing a potential TBI therapy in animal models have not adopted the gold standard required for clinical randomized controlled trials (RCTs) and often lack crucial features, such as randomization and masking.

Our study on the dose-response of Cerebrolysin in a rat model of mTBI was designed to take advantage of important clinical features of RCTs; therefore, our study was prospective, blinded, randomized, and placebo controlled. RCTs provide the highest level of evidence because they are designed to be unbiased and introduce less risk of systematic errors. This preclinical study was intended to find an optimal dose, and our results extend those in earlier reports by addressing the efficacy and dose-response effects of Cerebrolysin in a rat mTBI model. ${ }^{60}$ Furthermore, the combined 90-day end point reduced the potential for overinterpretation of the observed effect sizes. To eliminate any bias in animal selection and outcome measurement, we considered inclusion/exclusion criteria, sample-size calculation, randomization of animal treatment, predefined end points, and reporting of data analyses and data evaluations by a statistician who was independent of the research group and not involved in the animal work. The study medications (saline and Cerebrolysin) were prepared in opaque syringes at constant volumes by an independent investigator to ensure that the personnel involved in the experimental work were blinded to the treatment and dose. The global test on a battery of cognitive and sensorimotor functional outcomes was performed under careful statistical modeling and proper data transformation. The global test on multiple outcomes is more efficient than that on a single outcome when treatment effects are consistent, as previously shown in TBI and stroke research. ${ }^{37}$

Unlike patients with mTBI with a heterogeneous injury nature and population, our animal model of mTBI produced a relatively homogeneous type of brain injury in terms of persistent histological changes and cognitive deficits up to 3 months in the rats after injury. This model is very suitable for evaluating the efficacy of a treatment. ${ }^{39,57}$

\section{Study Limitations}

In this study, the initial Cerebrolysin treatment was applied to only 1 early time point (4 hours after injury) in the rats after mTBI. Results of our previous study also indicated that delayed treatment ( 24 hours after injury) improves functional recovery in rats after mTBI. ${ }^{60}$ Thus, results of our 2 studies indicate that early and delayed Cerebrolysin treatment is effective in rats after mTBI. TBI is a complex and chronic disease. Additional studies are warranted to assess the efficacy of early (within 4 hours) and late (beyond 24 hours) treatment with Cerebrolysin after mTBI.

It is important also to investigate the efficacy of Cerebrolysin in models of repeat mTBI. Increasing evidence has shown that repeated mTBI exaggerates functional deficits and delays recovery; ${ }^{20,30,53,56}$ repeat concussions often occur in contact sports.

Physiological features, including arterial blood pressure and blood gases $\left(\mathrm{pH}, \mathrm{PO}_{2}, \mathrm{PCO}_{2}\right)$, are important parameters to be monitored during experiments. These parameters were not measured in this study. Previous studies found that these physiological parameters are within the reference range for rats subjected to $\mathrm{mTBI}$ induced by impact acceleration. ${ }^{18,41}$ In addition, patient data from clinical trials have shown that there were no differences in physiological parameters and extensive hematological profile measurements between patient groups with stroke ${ }^{24,26,34}$ and those with $\mathrm{TBI}^{42}$ treated with Cerebrolysin or vehicle. Preclinical data are consistent with the absence of an effect of Cerebrolysin on physiological and hematological parameters in patients (EVER Pharma $\mathrm{GmbH}$, personal communication). Thus, it is likely that Cerebrolysin would not have affected the physiological parameters in the rats with mTBI used in our study. Nevertheless, it is important to include physiological monitoring in future studies.

Histological-functional correlations will strengthen the 
case for the efficacy of Cerebrolysin and will also help to delineate key mechanistic details. Because of space restrictions and the extensive histological data generated from this study, this article focuses on the dose-response of Cerebrolysin on functional outcomes. We plan to report the results of our extensive histological analysis in another publication. In brief, our histology data (in a separate report) reveal that Cerebrolysin treatment-induced improvement of functional recovery was significantly associated with increased neurogenesis in the dentate gyrus and decreased axonal damage, diffuse activation of astrocytes, and amyloid precursor protein accumulation in the brain after mTBI. These findings, in concert with those from previous preclinical and clinical studies,,$^{2,3,47}$ strongly indicate that Cerebrolysin is a promising therapy for mTBI.

It would be interesting to determine if there was even an acute cognitive deficit after mTBI, although contemporary literature suggests that cognitive deficits appear days or months after mTBI. ${ }^{13,60}$ In our study, we did not perform other measures (i.e., cognition, social interaction, and NOR tests) immediately after the mTBI because the early treatment initiated 4 hours after injury prevented us from performing these tests. In addition, the effects of anesthesia and surgical wounds confound performance in these tests.

\section{Clinical Translation}

The majority of clinical trials of TBI treated with Cerebrolysin used different doses, in the range of 10-50 $\mathrm{ml}$ for 5-21 days ${ }^{6}$ (e.g., $10 \mathrm{ml}$ for 30 days starting 1 month after severe TBI, ${ }^{31} 30 \mathrm{ml}$ for 5 days starting 24 hours after mTBI, ${ }^{8}$ and $10-50 \mathrm{ml}$ for 10 days starting 6 hours [acute] after TBI and repeated with the same treatment 30 days after injury for another 10 days if necessary, in a recent clinical design for Cerebrolysin treatment of severe TBI). ${ }^{47}$ We adapted the scheme of 10-90 ml for 10 days for our preclinical experiment to recapitulate RCTs, the gold standard for clinical trials, and facilitate the comparison with human data. The 0.8-, 2.5-, and 7.5-ml/kg intraperitoneal Cerebrolysin doses that were selected correspond to equivalent intravenous doses of 10,30, and $90 \mathrm{ml}$, respectively, in a 70- to 75$\mathrm{kg}$ human. Based on the observed dose-response, there was statistically significant separation of the 3 effective doses; higher doses produced better effects than the lower dose. However, we found no significant difference between the 2 higher doses. It is tempting to speculate that in humans, increased dosing would probably further improve neurological outcome and speed of recovery, which would help patients with mTBI return to their normal activities, and might facilitate early mobilization and early rehabilitation after severe TBI. Even the highest dose of $7.5 \mathrm{ml} / \mathrm{kg}$ (an approximately $90-\mathrm{ml}$ human dose) was well tolerated and did not result in any negative effect on mortality or animal health. Cerebrolysin was well tolerated, and no systemic pattern of toxicity was observed in patients with stroke even at a dose of $50 \mathrm{ml} /$ day for 21 days. ${ }^{33}$ It is important to note that the tests that are used in animal studies focus on sensorimotor skills and cognitive functions of the animals, whereas some tests in humans also incorporate more complex functions, such as speech and language abilities, drawing, verbal fluency, abstract thinking, and mental manipulation. ${ }^{8}$ Therefore, it is important not to overestimate the effect sizes in animal studies with respect to expectations in human trials. The results of a meta-analysis indicated that Cerebrolysin produces favorable results for the Glasgow Outcome Scale score and improvement of cognition in patients with TBI, although the numbers of subjects included in those studies were relatively small. ${ }^{15}$ In a double-blind, placebo-controlled, randomized study, patients with mTBI treated with Cerebrolysin within 24 hours of injury at a dose of $30 \mathrm{ml} /$ day for 5 days had enhanced cognitive recovery 3 months after injury. ${ }^{8}$ It should be noted that patients with mTBI suffered intracranial contusion hemorrhage, and the number of patients was small (15 for placebo and 17 for Cerebrolysin) in that trial. Additional double-blinded, randomized, multicenter clinical trials using Cerebrolysin with a larger number of patients are warranted.

\section{Outlook}

The results of our study show that Cerebrolysin significantly improves neurological outcomes in rats with mTBI in a dose-dependent manner when treatment is initiated 4 hours after injury. Using the same prospective, randomized, blinded, placebo-controlled protocol, the doseresponse effects of Cerebrolysin on functional outcomes were also shown in a rat model of stroke. ${ }^{59}$ Furthermore, our previous study found that delayed treatment with Cerebrolysin $(2.5 \mathrm{ml} / \mathrm{kg}$, starting 24 hours after injury and then daily for 28 days) improves cognitive function at least up to 3 months in a rat model of mTBI. ${ }^{60}$ Collectively, these findings suggest that Cerebrolysin has the potential to treat acute and subacute brain injuries. Clinically, pathophysiological heterogeneity of patients with TBI can arise from the primary injury (location, nature, and severity of injury) and preexisting conditions, including but not restricted to age, health, sex, medication, alcohol and drug use, and genetics, conditions that might also significantly affect the efficacy and safety of the treatment. ${ }^{57}$ Therefore, wide variability exists in the type of pathology and severity of injury in the clinical situation. Animal models of TBI produce a relatively homogeneous type of injury, with age, sex, genetic background, and injury parameters well controlled. ${ }^{57}$ However, none of the single-animal models perfectly mirror the complex conditions of TBI seen in human patients. This distinction might partially account for differences in TBI pathophysiology and therapeutic treatments between animal models and TBI clinical trials. Therefore, it will be important to further determine the efficacy of Cerebrolysin in multiple animal models of TBI and in both sexes and to include comorbidities and aged animals in rat models of TBI for our preclinical study of Cerebrolysin efficacy and safety.

\section{Acknowledgments}

We thank Susan MacPhee-Gray for her editorial assistance.

\section{References}

1. Alvarez XA, Cacabelos R, Sampedro C, Aleixandre M, Linares C, Granizo E, et al: Efficacy and safety of Cerebrolysin in moderate to moderately severe Alzheimer's disease: results of a randomized, double-blind, controlled trial investigating three dosages of Cerebrolysin. Eur J Neurol 18:59-68, 2011 
2. Alvarez XA, Sampedro C, Figueroa J, Tellado I, González A, García-Fantini M, et al: Reductions in qEEG slowing over 1 year and after treatment with Cerebrolysin in patients with moderate-severe traumatic brain injury. J Neural Transm (Vienna) 115:683-692, 2008

3. Alvarez XA, Sampedro C, Pérez P, Laredo M, Couceiro V, Hernández A, et al: Positive effects of cerebrolysin on electroencephalogram slowing, cognition and clinical outcome in patients with postacute traumatic brain injury: an exploratory study. Int Clin Psychopharmacol 18:271-278, 2003

4. Beaumont A, Marmarou A, Czigner A, Yamamoto M, Demetriadou K, Shirotani T, et al: The impact-acceleration model of head injury: injury severity predicts motor and cognitive performance after trauma. Neurol Res 21:742-754, 1999

5. Bengoetxea X, Rodriguez-Perdigon M, Ramirez MJ: Object recognition test for studying cognitive impairments in animal models of Alzheimer's disease. Front Biosci (Schol Ed) 7:10-29, 2015

6. Bornstein N, Poon WS: Accelerated recovery from acute brain injuries: clinical efficacy of neurotrophic treatment in stroke and traumatic brain injuries. Drugs Today (Barc) 48 (Suppl A):43-61, 2012

7. Centers for Disease Control and Prevention (CDC): Sportsrelated recurrent brain injuries-United States. MMWR Morb Mortal Wkly Rep 46:224-227, 1997

8. Chen CC, Wei ST, Tsaia SC, Chen XX, Cho DY: Cerebrolysin enhances cognitive recovery of mild traumatic brain injury patients: double-blind, placebo-controlled, randomized study. Br J Neurosurg 27:803-807, 2013

9. Chen J, Sanberg PR, Li Y, Wang L, Lu M, Willing AE, et al: Intravenous administration of human umbilical cord blood reduces behavioral deficits after stroke in rats. Stroke 32:2682-2688, 2001

10. Choi SH, Woodlee MT, Hong JJ, Schallert T: A simple modification of the water maze test to enhance daily detection of spatial memory in rats and mice. J Neurosci Methods 156:182-193, 2006

11. Crawley JN: Designing mouse behavioral tasks relevant to autistic-like behaviors. Ment Retard Dev Disabil Res Rev 10:248-258, 2004

12. Daneshvar DH, Riley DO, Nowinski CJ, McKee AC, Stern RA, Cantu RC: Long-term consequences: effects on normal development profile after concussion. Phys Med Rehabil Clin N Am 22:683-700, 2011

13. Darwish H, Mahmood A, Schallert T, Chopp M, Therrien B: Mild traumatic brain injury (MTBI) leads to spatial learning deficits. Brain Inj 26:151-165, 2012 (Erratum in Brain Inj 26:1763, 2012)

14. Dewitt DS, Perez-Polo R, Hulsebosch CE, Dash PK, Robertson CS: Challenges in the development of rodent models of mild traumatic brain injury. J Neurotrauma 30:688-701, 2013

15. El Sayed I, Zaki A, Fayed AM, Shehata GM, Abdelmonem S: A meta-analysis of the effect of different neuroprotective drugs in management of patients with traumatic brain injury. Neurosurg Rev [epub ahead of print], 2016

16. Elder GA, Dorr NP, De Gasperi R, Gama Sosa MA, Shaughness MC, Maudlin-Jeronimo E, et al: Blast exposure induces post-traumatic stress disorder-related traits in a rat model of mild traumatic brain injury. J Neurotrauma 29:2564-2575, 2012

17. Foda MA, Marmarou A: A new model of diffuse brain injury in rats. Part II: Morphological characterization. J Neurosurg 80:301-313, 1994

18. Fujita M, Wei EP, Povlishock JT: Intensity- and interval-specific repetitive traumatic brain injury can evoke both axonal and microvascular damage. J Neurotrauma 29:2172-2180, 2012

19. Gao X, Chen J: Mild traumatic brain injury results in exten- sive neuronal degeneration in the cerebral cortex. J Neuropathol Exp Neurol 70:183-191, 2011

20. Goddeyne C, Nichols J, Wu C, Anderson T: Repetitive mild traumatic brain injury induces ventriculomegaly and cortical thinning in juvenile rats. J Neurophysiol 113:3268-3280, 2015

21. Gonzales EL, Yang SM, Choi CS, Mabunga DF, Kim HJ, Cheong JH, et al: Repeated neonatal propofol administration induces sex-dependent long-term impairments on spatial and recognition memory in rats. Biomol Ther (Seoul) 23:251260,2015

22. Hallam TM, Floyd CL, Folkerts MM, Lee LL, Gong QZ, Lyeth BG, et al: Comparison of behavioral deficits and acute neuronal degeneration in rat lateral fluid percussion and weight-drop brain injury models. J Neurotrauma 21:521539,2004

23. Hawryluk GW, Bullock MR: Past, present, and future of traumatic brain injury research. Neurosurg Clin N Am 27:375-396, 2016

24. Heiss WD, Brainin M, Bornstein NM, Tuomilehto J, Hong Z: Cerebrolysin in patients with acute ischemic stroke in Asia: results of a double-blind, placebo-controlled randomized trial. Stroke 43:630-636, 2012

25. Hernandez TD, Schallert T: Seizures and recovery from experimental brain damage. Exp Neurol 102:318-324, 1988

26. Hong Z, Moessler H, Bornstein N, Brainin M, Heiss WD: A double-blind, placebo-controlled, randomized trial to evaluate the safety and efficacy of Cerebrolysin in patients with acute ischaemic stroke in Asia-CASTA. Int J Stroke 4:406-412, 2009

27. Howard RB, Sayeed I, Stein DG: Suboptimal dosing parameters as possible factors in the negative Phase III clinical trials of progesterone for traumatic brain injury. J Neurotrauma 34:1915-1918, 2017

28. Janowitz T, Menon DK: Exploring new routes for neuroprotective drug development in traumatic brain injury. Sci Transl Med 2:27rv1, 2010

29. Johnstone VP, Shultz SR, Yan EB, O'Brien TJ, Rajan R: The acute phase of mild traumatic brain injury is characterized by a distance-dependent neuronal hypoactivity. J Neurotrauma 31:1881-1895, 2014

30. Kamins J, Giza CC: Concussion-mild traumatic brain injury: recoverable injury with potential for serious sequelae. Neurosurg Clin N Am 27:441-452, 2016

31. Khalili H, Niakan A, Ghaffarpasand F: Effects of cerebrolysin on functional recovery in patients with severe disability after traumatic brain injury: a historical cohort study. Clin Neurol Neurosurg 152:34-38, 2017

32. Kilkenny C, Browne W, Cuthill IC, Emerson M, Altman DG: Animal research: reporting in vivo experiments: the ARRIVE guidelines. J Gene Med 12:561-563, 2010

33. Ladurner G, Kalvach P, Moessler H: Neuroprotective treatment with cerebrolysin in patients with acute stroke: a randomised controlled trial. J Neural Transm (Vienna) 112:415-428, 2005

34. Lang W, Stadler CH, Poljakovic Z, Fleet D: A prospective, randomized, placebo-controlled, double-blind trial about safety and efficacy of combined treatment with alteplase (rtPA) and Cerebrolysin in acute ischaemic hemispheric stroke. Int J Stroke 8:95-104, 2013

35. Loane DJ, Faden AI: Neuroprotection for traumatic brain injury: translational challenges and emerging therapeutic strategies. Trends Pharmacol Sci 31:596-604, 2010

36. Lu J, Gary KW, Neimeier JP, Ward J, Lapane KL: Randomized controlled trials in adult traumatic brain injury. Brain Inj 26:1523-1548, 2012

37. Lu M, Chen J, Lu D, Yi L, Mahmood A, Chopp M: Global test statistics for treatment effect of stroke and traumatic brain injury in rats with administration of bone marrow stromal cells. J Neurosci Methods 128:183-190, 2003 
38. Mahmood A, Goussev A, Lu D, Qu C, Xiong Y, Kazmi $\mathrm{H}$, et al: Long-lasting benefits after treatment of traumatic brain injury (TBI) in rats with combination therapy of marrow stromal cells (MSCs) and simvastatin. J Neurotrauma 25:1441-1447, 2008

39. Marklund N, Hillered L: Animal modelling of traumatic brain injury in preclinical drug development: where do we go from here? Br J Pharmacol 164:1207-1229, 2011

40. Marmarou A, Foda MA, van den Brink W, Campbell J, Kita $\mathrm{H}$, Demetriadou K: A new model of diffuse brain injury in rats. Part I: Pathophysiology and biomechanics. J Neurosurg 80:291-300, 1994

41. Miyauchi T, Wei EP, Povlishock JT: Evidence for the therapeutic efficacy of either mild hypothermia or oxygen radical scavengers after repetitive mild traumatic brain injury. J Neurotrauma 31:773-781, 2014

42. Muresanu DF, Ciurea AV, Gorgan RM, Gheorghita E, Florian SI, Stan H, et al: A retrospective, multi-center cohort study evaluating the severity- related effects of cerebrolysin treatment on clinical outcomes in traumatic brain injury. CNS Neurol Disord Drug Targets 14:587-599, 2015

43. Muresanu DF, Heiss WD, Hoemberg V, Bajenaru O, Popescu CD, Vester JC, et al: Cerebrolysin and Recovery After Stroke (CARS): a randomized, placebo-controlled, double-blind, multicenter trial. Stroke 47:151-159, 2016

44. Narayan RK, Michel ME, Ansell B, Baethmann A, Biegon A, Bracken MB, et al: Clinical trials in head injury. J Neurotrauma 19:503-557, 2002

45. Newcombe VF, Menon DK: Cognitive deficits and mild traumatic brain injury. BMJ 346:f1522, 2013

46. Plosker GL, Gauthier S: Cerebrolysin: a review of its use in dementia. Drugs Aging 26:893-915, 2009

47. Poon W, Vos P, Muresanu D, Vester J, von Wild K, Hömberg $\mathrm{V}$, et al: Cerebrolysin Asian Pacific trial in acute brain injury and neurorecovery: design and methods. J Neurotrauma 32:571-580, 2015

48. Semple BD, Canchola SA, Noble-Haeusslein LJ: Deficits in social behavior emerge during development after pediatric traumatic brain injury in mice. J Neurotrauma 29:26722683, 2012

49. Sharma HS, Zimmermann-Meinzingen S, Johanson CE: Cerebrolysin reduces blood-cerebrospinal fluid barrier permeability change, brain pathology, and functional deficits following traumatic brain injury in the rat. Ann N Y Acad Sci 1199:125-137, 2010

50. Shen LH, Li Y, Chen J, Cui Y, Zhang C, Kapke A, et al: Oneyear follow-up after bone marrow stromal cell treatment in middle-aged female rats with stroke. Stroke 38:2150-2156, 2007

51. Stuart SA, Robertson JD, Marrion NV, Robinson ES: Chronic pravastatin but not atorvastatin treatment impairs cognitive function in two rodent models of learning and memory. PLoS One 8:e75467, 2013

52. Tay SY, Ang BT, Lau XY, Meyyappan A, Collinson SL: Chronic impairment of prospective memory after mild traumatic brain injury. J Neurotrauma 27:77-83, 2010

53. Thomsen GM, Ma AM, Ko A, Harada MY, Wyss L, Haro PS, et al: A model of recurrent concussion that leads to long-term motor deficits, CTE-like tauopathy and exacerbation of an ALS phenotype. J Trauma Acute Care Surg 81:1070-1079, 2016

54. Ubhi K, Rockenstein E, Doppler E, Mante M, Adame A, Patrick C, et al: Neurofibrillary and neurodegenerative pathology in APP-transgenic mice injected with AAV2-mutant TAU: neuroprotective effects of Cerebrolysin. Acta Neuropathol 117:699-712, 2009
55. US Food and Drug Administration: Guidance for Industry: Estimating the Maximum Safe Starting Dose in Initial Clinical Trials for Therapeutics in Adult Healthy Volunteers. Rockville, MD: US Food and Drug Administration, 2005 (https://www.fda.gov/downloads/drugs/guidances/ ucm078932.pdf) [Accessed August 30, 2017]

56. Vagnozzi R, Tavazzi B, Signoretti S, Amorini AM, Belli A, Cimatti M, et al: Temporal window of metabolic brain vulnerability to concussions: mitochondrial-related impairment-part I. Neurosurgery 61:379-389, 2007

57. Xiong Y, Mahmood A, Chopp M: Animal models of traumatic brain injury. Nat Rev Neurosci 14:128-142, 2013

58. Zhang C, Chopp M, Cui Y, Wang L, Zhang R, Zhang L, et al: Cerebrolysin enhances neurogenesis in the ischemic brain and improves functional outcome after stroke. J Neurosci Res 88:3275-3281, 2010

59. Zhang L, Chopp M, Lu M, Zhang T, Winter S, Doppler E, et al: Cerebrolysin dose-dependently improves neurological outcome in rats after acute stroke: A prospective, randomized, blinded, and placebo-controlled study. Int J Stroke 11:347-355, 2016

60. Zhang Y, Chopp M, Meng Y, Zhang ZG, Doppler E, Winter $\mathrm{S}$, et al: Cerebrolysin improves cognitive performance in rats after mild traumatic brain injury. J Neurosurg 122:843-855, 2015

61. Zhang Y, Zhang ZG, Chopp M, Meng Y, Zhang L, Mahmood A, et al: Treatment of traumatic brain injury in rats with $\mathrm{N}$-acetyl-seryl-aspartyl-lysyl-proline. J Neurosurg 126:782795,2017

\section{Disclosures}

Drs. Winter and Brandstätter are employees of Clinical Research and Pharmacology, EVER Neuro Pharma GmbH (Unterach, Austria), which did not participate in the animal studies, data collection, or data analyses. All the authors designed the study together, and EVER Neuro reviewed the report and manuscript without interfering with the conclusions that were drawn. This work was supported by EVER Pharma GmbH.

\section{Author Contributions}

Conception and design: Xiong, Chopp, Gang Zhang. Acquisition of data: Xiong, Yanlu Zhang. Analysis and interpretation of data: Xiong, Chopp, Gang Zhang, Lu, T Zhang. Drafting the article: Xiong, Yanlu Zhang. Critically revising the article: Xiong, Chopp, Gang Zhang, L Zhang, Winter, Brandstätter, Mahmood. Reviewed submitted version of manuscript: all authors. Approved the final version of the manuscript on behalf of all authors: Xiong. Administrative/technical/material support: Yi Zhang. Preparation of medication: Yi Zhang.

\section{Supplemental Information \\ Online-Only Content}

Supplemental material is available with the online version of the article.

Supplemental Information. https://thejns.org/doi/suppl/10. 3171/2017.6.JNS171007.

\section{Correspondence}

Ye Xiong: Henry Ford Health System, Detroit, MI. yxiong1@ hfhs.org. 